\title{
INVESTIGAÇÃO DA ATIVIDADE ANTIFÚNGICA DE Mangifera indica L. FRENTE A DIFERENTES ESPÉCIES DE Candida sSp. ASSOCIADAS À CANDIDÍASE VULVOVAGINAL
}

\author{
Karla Maria Santos de Oliveira ${ }^{1}$ \\ Renata Soares da Silva ${ }^{1}$ \\ Giani Maria Cavalcante ${ }^{*}$
}

OLIVEIRA, K. M. S. de; SILVA, R. S. da; CAVALCANTE, G. M. Investigação da atividade antifúngica de Mangifera indica L. frente a diferentes espécies de Candida ssp. associadas à candidíase vulvovaginal. Arq. Cienc. Saúde UNIPAR, Umuarama, v. 19, n. 3, p, 179183, set/dez. 2015.

RESUMO: Candidíase Vaginal é a infecção fúngica oportunista mais comum e importante nas mulheres. O aumento na utilização de drogas antifúngicas, nos últimos anos, tem causado resistência aos medicamentos disponíveis para o tratamento. O objetivo deste estudo foi investigar a atividade antifúngica de Mangifera indica em estirpes associadas à candidíase vaginal. Os testes in vitro foram realizados com o extrato bruto e frações orgânicas contra as estirpes de Candida albicans (URM 4385), Candida glabrata (URM 4264) e Candida tropicalis (URM 4262), gentilmente cedidas pela Micoteca da Universidade Federal de Pernambuco (UFPE), por meio do ensaio de difusão em ágar por meio de poço e determinação da concentração inibitória mínima (CIM). O extrato etanólico foi o mais ativo, com diâmetro de inibição variando entre 25,5 e 18,5 mm, valores semelhantes à droga padrão, não apresentando diferença estatística. A CIM variou de 0,04 e $0,16 \mathrm{mg} / \mathrm{ml} \mathrm{em} \mathrm{microrganismo} \mathrm{testado.} \mathrm{As} \mathrm{frações} \mathrm{acetato} \mathrm{de} \mathrm{etila} \mathrm{e} \mathrm{metanólica} \mathrm{apresentaram} \mathrm{atividade} \mathrm{antifúngica} \mathrm{relevante} \mathrm{contra} C$. glabrata e C. albicans, respectivamente. Estudo químico para a extração e o isolamento dos compostos ativos é recomendado para ensaios in vitro destes compostos para investigar a sua atividade antifúngica.

PALAVRAS-CHAVE: Mangifera indica; Candidíase Vaginal; Atividade antifúngica.

\section{RESEARCH OF Mangifera indica L. ANTIFUNGAL ACTIVITY IN DIFFERENT Candida SSP. SPECIES ASSOCIATED WITH VULVOVAGINAL CANDIDIASIS}

\begin{abstract}
Vaginal Candidiasis is the most common and important opportunistic fungal infection in women. The increased use of antifungal drugs in recent years has caused resistance to the drugs available for treatment. The aim of this study was to investigate the antifungal activity of Mangifera indica on strains associated to vaginal candidiasis. In-vitro tests were conducted with crude extract and organics fractions against strains of Candida albicans (URM 4385), Candida glabrata (URM 4264) and Candida tropicalis (URM 4262), a courtesy of the Federal University of Pernambuco (UFPE), through diffusion in agar-bem assay and minimal inhibitory concentrations (MIC). The ethanolic extract was the most active, with inhibition diameter ranging between 25.5 to $18.5 \mathrm{~mm}$, values that are similar to the standard drug, not presenting statistic different. The MIC varied from 0.04 to $0.16 \mathrm{mg} / \mathrm{ml}$ for the tested microorganism. The ethyl and methanolic acetate organic fractions presented relevant antifungal activity against $C$. glabrata and C. albicans, respectively. The chemical study for the extraction and isolation of active compounds is recommended for in-vitro assays of these compounds to investigate the antimicrobial activity.
\end{abstract}

KEYWORDS: Mangifera indica; Vaginal Candidiasis; Antifungal activity.

\section{Introdução}

O gênero Candida é constituído por aproximadamente 200 espécies de leveduras, ocorrentes em diferentes nichos corporais (região vulvovaginal, orofaringe, cavidade bucal, secreções brônquicas), as espécies pertencentes a esse gênero são classificadas como patógenos oportunistas e frequentemente estão associadas à infecções endógenas (ÁLVARES; SVIDZINSKI; CONSOLARO, 2007).

A candidíase vulvovaginal (CVV) é uma infecção da vulva e da vagina ocasionada pelas espécies de Candida sp. que compõem a flora vaginal e digestiva e que crescem em meio favorável ao seu desenvolvimento (BOATTO et al., 2015). Embora não seja uma infecção que proporcione grande impacto na saúde pública, a CVV é classificada como a mais comum vaginite aguda nos países tropicais, e a sua incidência varia de $25 \%$ na população feminina em geral a $42 \%$ em mulheres adolescentes, havendo uma população de risco de infecção em torno de $75 \%$.

Segundo Galle e Gianinni (2004) as espécies de
Candida prevalentes em CVV são Candida albicans, Candida glabrata e Candida tropicalis, com registros de ocorrência de $74 \%, 14 \%$ e $7 \%$, respectivamente, em culturas de fluido vaginal. Enquanto, em culturas teciduais, foram registradas ocorrências de $86 \%$ para C. albicans, $11 \%$ para C. glabrata e $5 \%$ para C. tropicalis.

O tratamento da CVV inclui agentes antimicóticos de uso tópico ou sistêmico da classe dos azóis, baseado nos núcleos imidazol ou trizol. Esse agente com ação fungistática apresenta um amplo espectro de atividade (BEZERRA et al., 2015). Entretanto, a susceptibilidade das várias espécies de Candida para os antifúngicos mais usados e a resistência intrínseca frente à terapêutica antifúngica, tem ocasionado o desenvolvimento de resistência pelas espécies de Candida e está se tornando um grande problema no tratamento da infecção (MOHAMADI et al., 2015; SANGUINETTI; POSTERARO; LASS-FLORL, 2015).

Pfaller; Diekema (2007) registraram resistência cruzada entre azoles durante ensaios in vitro entre as espécies de Candida associdas a CVV. Enquanto, a pesquisa de Lopez-

DOI: https://doi.org/10.25110/arqsaude.v19i3.2015.5234

${ }^{1}$ Estudante de Graduação em Enfermagem do Centro Universitário Cesmac.

${ }^{2}$ Professora do Centro Universitário Cesmac.

*Endereço para correspondência: Rua Cônego Machado, 918 - Farol, Maceió - AL, 57051-160. Telefone: +55 82 3215-5000. Email: gianimc@icloud.com 
-Ribot; Mcatee; Lee (1998) detectou susceptibilidade reduzida a azoles em isolados de Candida oriundos de pacientes com infecção grave.

Neste contexto, torna-se relevante a busca por meios terapêuticos alternativos para o tratamento da CVV tendo em vista a elevada frequência e importância clínica atribuída à patologia. Partindo dessa necessidade foi investigada a atividade antifúngica de Mangifera indica frente às espécies de Candida spp. associadas à CVV.

Mangifera indica L. é uma espécie vegetal da família Anacardiaceae cujo arsenal terapêutico registra atividade antimicrobiana (GARRIDO et al., 2001), efeitos analgésicos, anti-inflamatórios e antioxidante (ENGELS; SCHIEBER; GANZLE, 2011) e atividade antidiarreica (KASSI et al., 2014).

Embora tenha sido encontrado registro de atividade antifúngica de extratos de $M$. indica (KANWAL et al., 2010), as informações são escassas, em especial para as espécies prevalentes em CVV. Sendo assim, o objetivo deste trabalho foi investigar a atividade antifúngica in vitro do extrato bruto de folhas e frações orgânicas de Mangifera indica L. sob o desenvolvimento de espécies associadas à candidíase vulvovaginal.

\section{Materiais e Métodos}

A espécie vegetal foi obtida junto ao viveiro do Centro de Ciências Agrárias da Universidade Federal de Alagoas (CECA-UFAL). Partes vegetais (folha, flor e talo) foram acondicionadas em sacos plásticos individuais e levadas ao Herbário do CECA-UFAL, para confirmação da espécie junto a profissionais de botânica. Uma exsicata foi confeccionada e guardada sob o ${ }^{\circ}$ IMAL-9848.

O extrato etanólico (EEtOH) e as frações orgânicas Hexânica (FrHex), Metanólica (FrMeOH) e Acetato de Etila (AcEOt), oriundos das folhas de $M$. indica, foram gentilmente cedidos pelo Laboratório de Fitoquímica do Centro de Ciências Agrárias da Universidade Federal de Alagoas. Para os ensaios de atividade antifúngica o extrato bruto e as frações orgânicas foram previamente solubilizados em dimetilsulfóxido (DMSO), uma vez que este não interfere no desenvolvimento de microrganismos patogênicos (OSTROSKY et al., 2008).

Os microrganismos Candida albicans (URM 4385), Candida glabrata (URM 4264) e Candida tropicalis (URM 4262), usados nesta pesquisa, foram gentilmente cedidos pela Micoteca do Departamento de Micologia da Universidade Federal de Pernambuco (UFPE).

Todos os microrganismos foram mantidos em meio ágar Sabouraud a uma temperatura de $4{ }^{\circ} \mathrm{C}$, até a sua utilização nos ensaios antifúngicos. Quando selecionados para os ensaios, os microrganismos foram inoculados, com auxílio de $s w a b$, em placas de Petri contendo meio ágar Sabouraud dextrose e incubados a $37{ }^{\circ} \mathrm{C}$ por $24 \mathrm{~h}$ para crescimento dos mesmos. Em seguida as suspensões foram preparadas em solução salina estéril ( $\mathrm{NaCl}$ a $0,85 \%)$ e padronizadas por comparação com o tubo 1.0 da escala de MacFarland, conforme metodologia descrita por Essama et al. (2015).

Inicialmente, a atividade antifúngica foi determinada por meio do ensaio de difusão em ágar por meio de poço conforme a metodologia descrita por Nennaah (2013). Em placas de Petri contendo o meio ágar Sabouraud dextrose as espécies de Candida foram inoculadas, separadamente, com o auxílio de um $S w a b$; em seguida quatro cavidades (6 mm de diâmetro) foram feitas usando pipetas de Pasteur estéreis. Os poços foram saturados, separadamente, com extrato bruto e frações orgânicas em concentrações variando de 400 a $100 \mu \mathrm{g} / \mathrm{mL}$. As placas foram incubadas a $28^{\circ} \mathrm{C}$ por $48 \mathrm{~h}$. Os diâmetros das zonas de inibição foram medidos com o auxílio de uma escala milimétrica. Os testes foram realizados em duplicatas.

A determinação da Concentração Inibitória Mínima (CIM) foi realizada pelo método de microdiluição em placa de 96 poços de acordo com as normas do protocolo NCCLS (M27-A2). A concentração inicial do extrato bruto foi de 400 $\mu \mathrm{g} / \mathrm{mL}$ e nos poços seguintes foi adicionado meio de cultura (Ágar Sabouroud Dextrose) de forma que a concentração diminuísse de 50 em $50 \mu \mathrm{g} / \mathrm{mL}$, até a concentração de $100 \mu \mathrm{g} /$ mL (ESSAMA et al., 2015). Como controles foram usados uma solução contendo $20 \mu \mathrm{L}$ de suspensão e $80 \mu \mathrm{L}$ de meio de cultivo líquido (C1) e uma solução contendo $20 \mu \mathrm{L}$ de suspensão, $70 \mu \mathrm{L}$ de meio de cultivo líquido e $10 \mu \mathrm{L}$ do antifúngico intraconazol na concentração de $50 \mu \mathrm{g} / \mu \mathrm{L}$ (C2), conforme descrito por Anthikat et al. (2014). Em seguida, as placas foram incubadas em estufas a $37{ }^{\circ} \mathrm{C}$ por $24 \mathrm{~h}$. Após esse período foi realizada a leitura utilizando um leitor de ELISA a $595 \mathrm{~nm}$. Os testes foram realizados em triplicatas.

Os dados obtidos no ensaio de difusão em ágar por meio de poço (diâmetro de inibição) foram expressos como media \pm desvio padrão $(\mathrm{X} \pm \mathrm{DP})$ e as médias foram analisadas estatisticamente usando análise de variância (ANOVA), comparadas pelo teste de Tukey usando o software Bioestat 5.0. As Diferenças foram consideradas significadas quando $\mathrm{p}>0,05$.

\section{Resultados}

Os resultados mais promissores para atividade antifúngica do extrato EEtOH e das frações orgânicas de $M$. indica frente a todas as espécies de Candida spp. testadas foram registrados na concentração de $150 \mu \mathrm{g} / \mathrm{mL}$ (Tabela 1). 
Tabela 1: Atividade antifúngica do extrato etanólico e das frações orgânicas de Mangifera indica $(150 \mu \mathrm{g} / \mathrm{mL})$ frente a diferentes espécies de Candida ssp. associadas à candidíase vulvovaginal. EEtOH (Extrato Etanólico), FrHex (Fração Hexânica), FrMeOH (Fração Metanólica), FrAcEOt (Fração Acetato), C1 (Suspensão antifúgica + meio de cultura), C2 (antifúngico intraconazol).

\begin{tabular}{|c|c|c|}
\hline Cepa bacteriana & Tratamentos & Médias da Zona de Inibição $(\mathrm{mm} \pm \mathrm{DP})$ \\
\hline \multirow{6}{*}{ C.albicans (URM 4385), } & EEtOH & $24,0 \pm 1,0^{\mathrm{a}}$ \\
\hline & FrHex & $18,0 \pm 1,0^{\mathrm{b}}$ \\
\hline & $\mathrm{FrMeOH}$ & $16,5 \pm 0,5^{\circ}$ \\
\hline & FrAcEOt & $22,5 \pm 0,5^{\mathrm{a}}$ \\
\hline & $\mathrm{C} 1$ & NA \\
\hline & $\mathrm{C} 2$ & $25,0 \pm 0,5^{\mathrm{a}}$ \\
\hline \multirow{6}{*}{ C. glabrata (URM 4264) } & EEtOH & $18,5 \pm 0,5^{\mathrm{a}}$ \\
\hline & FrHex & $12,5 \pm 1,0^{c}$ \\
\hline & $\mathrm{FrMeOH}$ & $19,5 \pm 0,5^{\mathrm{a}}$ \\
\hline & FrAcEOt & $14,0 \pm 0,5^{\mathrm{b}}$ \\
\hline & $\mathrm{C} 1$ & NA \\
\hline & $\mathrm{C} 2$ & $19,0 \pm 0,5^{\mathrm{a}}$ \\
\hline \multirow{6}{*}{ C.tropicalis (URM 4262), } & EEtOH & $24,5 \pm 1,0^{\mathrm{a}}$ \\
\hline & FrHex & $12,0 \pm 0,7^{\mathrm{d}}$ \\
\hline & FrMeOH & $18,5 \pm 1,0^{\mathrm{b}}$ \\
\hline & FrAcEOt & $14,0 \pm 0,5^{\circ}$ \\
\hline & $\mathrm{C} 1$ & $\mathrm{NA}$ \\
\hline & $\mathrm{C} 2$ & $25,0 \pm 0,5^{\mathrm{a}}$ \\
\hline
\end{tabular}

NA (Não ativo)

$\mathrm{Na}$ mesma coluna, médias seguidas por letras iguais não apresentam diferença estatística significativa $(\mathrm{p} \leq 0,05)$.

A concentração inibitória mínima variou entre 0,04 e $0,32 \mathrm{mg} / \mathrm{mL}$, sendo os menores valores registrados para EEtOH (Tabela 2).

Tabela 2: Concentração Inibitória Mínima (CIM)do extrato etanólico e das frações orgânicas de Mangifera indica $(150 \mu \mathrm{g} /$ $\mathrm{mL})$ frente a diferentes espécies de Candida ssp. associadas à candidíase vulvovaginal. EEtOH (Extrato Etanólico), FrHex (Fração Hexânica), FrMeOH (Fração Metanólica), FrAcEOt (Fração Acetato), C1 (Suspensão antifúgica + meio de cultura), C2 (antifúngico intraconazol).

\begin{tabular}{|c|c|c|}
\hline Cepa bacteriana & Tratamentos & MIC $(\mathrm{mg} / \mathrm{mL})$ \\
\hline \multirow{6}{*}{ C.albicans (URM 4385) } & EEtOH & 0,08 \\
\hline & FrHex & 0,32 \\
\hline & FrMeOH & 0,04 \\
\hline & FrAcEOt & 0,16 \\
\hline & $\mathrm{Cl}$ & $\mathrm{NA}$ \\
\hline & $\mathrm{C} 2$ & 0,125 \\
\hline \multirow{6}{*}{ C.glabrata (URM 4264) } & EEtOH & 0,16 \\
\hline & FrHex & 0,32 \\
\hline & $\mathrm{FrMeOH}$ & 0,32 \\
\hline & FrAcEOt & 0,64 \\
\hline & $\mathrm{C} 1$ & NA \\
\hline & $\mathrm{C} 2$ & 0,5 \\
\hline \multirow{6}{*}{ C.tropicalis (URM 4262) } & EEtOH & 0,04 \\
\hline & FrHex & 0,16 \\
\hline & FrMeOH & 0,16 \\
\hline & FrAcEOt & 0,32 \\
\hline & $\mathrm{Cl}$ & $\mathrm{NA}$ \\
\hline & $\mathrm{C} 2$ & 0,5 \\
\hline
\end{tabular}

$\mathrm{NA}=$ Não ativo

\section{Discussão}

Sabe-se que um dos principais fatores que dificultam o tratamento de infecções fúngicas é a resistência desenvolvida pelos microrganismos frente aos agentes antifúngicos sintéticos disponíveis (MARTINS et al., 2015). Em virtude do potencial terapêutico de produtos naturais, desvendado dia-a-dia, em especial a atividade antimicrobiana, a potencialidade efetiva de descobertas de novos compostos eficazes na prevenção e tratamento de doenças infecciosas tem aumento substancialmente.

Nesta pesquisa o extrato etanólico das folhas de $M$. indica se apresentou com elevado potencial antifúngico frente às espécies $C$. albicans, $C$. glabrata e $C$. tropicalis no que concerne a inibição do crescimento, com valores de concentrações inibitórias mínimas de 0,08, 0,16 e 0,04 mg/mL, respectivamente. Por sua vez as frações orgânicas, apresentaram atividade antimicrobiana intermediária, o que estimula a investigação acerca da atividade antifúngica de compostos isolados oriundos do extrato EEtOH e das frações orgânicas FrHex, FrMeOHeAcEOt, frente a esses microrganismos.

$\mathrm{O}$ arsenal antimicrobiano de $M$. indica inclui atividade inibitória sob o crescimento das espécies Bacillus subtilis, Bacillus cereus, Clostridium botulinum, Campylobacter jejuni, Listeria monocytogenes e Staphylococcus aureus dos compostos isolados enta-, hexa-, hepta-, octa-, nona-, and deca-O-galloylglucose (ENGELS; SCHIEBER; GANZLE, 2011). Singh et al. (2009) registraram atividade antimicrobiana do composto mangerin, isolado de caule, e seus análogos sintéticos frente às espécies Staphylococcus aureus, Escherichia coli e Aspergillus niger. Esses mesmos autores apontam que o análogo sintético de mangerin, 5-(N- $\alpha-$ napthylaminomethyleno), inibiu o crescimento da espécie C. albicans, entre tanto não foi testado a CIM. Segundo Ostrosky et al. (2008) determinar a concentração inibitória mínima é um aspecto relevante na pesquisa com substâncias e compostos naturais, uma vez que a CIM determinar a menor quantidade de substância necessária para inibir o crescimento do microrganismo e dessa forma avaliar aspectos toxicológicos, microbiológicos e legais pertinentes aos compostos naturais, suas combinações e seus derivados.

No tocante a atividade antifúngica de $M$. indica, poucos trabalhos foram encontrados, o mais relevante é o de Parekh; Chanda (2008), que registrou halo de inibição para extrato metanólico das folhas frente às espécies $C$. albicans (10 mm) e C. glabrata (9 mm), em uma concentração de 150 $\mu \mathrm{g} / \mathrm{mL}$. Os mesmos autores não registraram atividade dos extratos de $M$. indica para a espécie $C$. tropicalis. Ao comparar os resultados dos referidos autores com os obtidos nesta pesquisa, alguns aspectos relevantes precisam ser notificados: (a) as cepas utilizadas para investigação são diferentes, os autores supracitados usaram cepas da National Chemical Laboratory (NCIM), o que pode inferir nos resultados obtidos; (b) os autores registram atividade intermediária em uma concentração de $125 \mu \mathrm{g} / \mathrm{mL}$, enquanto nessa pesquisa os melhores resultados registrados foram na concentração de 150 $\mu \mathrm{g} / \mathrm{mL}$, a comparação dos dados aponta a possibilidade de reduzir a concentração dos extratos e frações ora testadas; (c) embora os autores não tenham registrado atividade de $M$. indica frente a C. tropicalis, nesta pesquisa o EEtOH apresentou atividade antifúngica similar ao antifúngico padrão, 
não diferindo estatisticamente deste, com a halo de inibição igual a 24,5 mm e CIM de 0,04.

Diante dos resultados de atividade antifúngica apresentados pelo extrato etanólico e frações orgânicas de $M$. indica fica evidente a necessidade de um estudo químico acerca de extração e isolamento de compostos que viabilizem estudos in vitro da atividade antimicrobiana desses frente ao crescimento de espécies fúngicas associadas a CVV.

\section{Conclusão}

Os resultados sugerem que Mangífera indica pode ser um promissor antifúngico de origem natural para o tratamento de infecções fúngicas ocorrentes em tecidos vulvovaginal.

Em virtudes de resultados promissores do extrato etanólico e das frações orgânicas hexanica, metanólica e acetato de etila, sugere-se estudo químico detalhado para obtenção de compostos isolados para testes antifúngicos frente a espécies ocorrentes em candidíase vulvovaginal.

\section{Referências}

ÁlVARES, C. A.; SVIDZINSKI, T. I.; CONSOLARO, M. E. L. Candidíase vulvovaginal: fatores predisponentes do hospedeiro e virulência das leveduras. Jornal Brasileiro de Patologia e Medicina Laboratorial, v.43, n.5, p.319-327, 2007.

ANTHIKAT, R. R. N. et al. Antifungal activity of Areca catechu L. International Journal of Pharmaceutical and Clinic Science, v.4, n.1, p.1-3, 2014.

BEZERRA, K. K. S. et al. Profile and susceptibility to vaginal yeast of women meto $n$ a gynecology out patient clinic. International Archives of Medicine, v.8, n.145, p.1-6, 2015.

BOATTO, H. F. et al. O papel dos parceiros sexuais sintomáticos e assintomáticos nas vulvovaginites recorrentes. Revista Brasileira de Ginecologia \& Obstetrícia, v.37, n.7, p.314-318, 2015.

ENGELS, C.; SCHIEBER, A.; GANZLE, M. G. Inhibitory spectra and modes of antimicrobial of gallotannins from mango kernels (Mangifera indica L.). Applied and Environmental Microbiology, v.77, n.7, p.2215-2223, 2011.

ESSAMA, S. H. R. et al.. In vitro evaluation of the antifungal activity of extracts of Baillonella toxisperma (Pierre), a sapotaceae, on the grownth of some nom an pathogenic yeasts. African Journal of Pharmacy and Pharmacology, v.9, n,9, p.299-306, 2015.

GALLE, L. C.; GIANINNI, M. J. S. M. Prevalência e susceptibilidade de leveduras vaginais. Jornal Brasileiro de Patologia e Medicina Laboratorial, v. 40, n.4, p. 229236, 2004.

GARRIDO, G. et al. Analgesicandanti-inflammatory effects of Mangifera indica extract. Phytotherapy Research, v.15, n.1, p. 18-21, 2001.

KANWAL, Q. et al. Antifungal activity of flavonoids isolated from mango (Mangifera indica L.) leaves. Natural Product Research: Formerly Natural Product Letters, v.24, n.10, p. 1907-1914, 2010.

KASSI, A. B. B. et al. Isolation and identification of bioactive compounds from kernel seed cake of the mango (Mangifera indica). International Journal Biological and Chemistry Science, v.8, n.4, p.1885-1895, 2014.

LOPEZ-RIBOT, J.L.; MCATEE, R.K.; LEE, L.N. Distinct patterns of gene expression associated with development of fluconazole resistance in serial Candida albicans isolates from human immunodeficiency virus infected patients with oropharyngeal candidiasis. Antimicrobiology Agents Chemotherapy, v.42, n.2, 2932-2937, 1998.

MOHAMADI, J. et al. Antifungal drug resistance pattern of Candida spp. isolated from vaginitis in Ilam-Iran during 2013-2014. Bioinformation, v.11, n.4, p.203-206, 2015.

MARTINS, N. et al. Plantas used in flok medicine: The potential of their hydromethanolic extracts against Candida species. Industrial Crops and Products, v.66, n.2, p.62-67, 2015.

NCCLS (Clinical and Laboratory Standards institute) Performace standards for antimicrobial disk susceptibility tests: approved standard. Wayne, Pennsylvania, 2003 (NCCLS document M2-A28 - ISBN 1-56238-485-6).

NENAAH, G. Antimicrobial activity of Calotropis proceraAit (Asclepiadaceae) and isolation of four flavonoid glycosides as the active constituents. World Journal Microbiology Biotechnology, v.29, n.1, p. 1255-1262, 2013.

OSTROSKY, E. A. et al. Métodos para avaliação da atividade antimicrobiana e determinação da concentração inibitória mínima (CIM) de plantas medicinais. Brazilian Journal of Pharmacognosy, v.18, n.2, p.301-307, 2008.

PAREKH, J.; CHANDA, S. In vitro antifungal activity of methanol extracts of some Indian medicinal plants against pathogenic yeast and moulds. African Journal of Biotechnology, v.7, n.23, p.4349-4353, 2008.

PFALLER, M. A.; DIEKMA, D. J. Epidemiology of invasive candidiasis: a persistent public health problem. Clinical Microbiology Reviews, v.20, n.1, p.133-163, 2007.

SANGUINETTI, M.; POSTERARO, B.; LASS-FLORL, C. Antifungal drug resistance among Candida species: mechanisms and clinical impact. Mycoses, v.58, n.2, p.2-13, 2015. 
Investigação da atividade antifúngica de Mangifera indica

SINGH, S.K. et al. Antimicrobial evaluation of Mangifera indica. Indian Journal of Pharmaceutical Sciences, v.71, n.3, p.328-331, 2009.

Recebido: 09/01/2015

Aceito: 30/11/2015 